\title{
CHARACTERISTICS OF SELECTED APPROACHES OF UNCERTAINTY MODELLING IN THE CONTEXT OF MANAGEMENT SCIENCES
}

\begin{abstract}
Information uncertainty in management systems is a subject of research of many scientific disciplines. Research concerning a quantitative modelling of uncertainty verging on mathematics, information technology, and logic is extremely important. The purpose of the article is to present possibilities and limitations of the most popular approaches in uncertainty modelling in social sciences with particular emphasis on management problems. Achieving such a goal always requires accepting certain basic epistemological assumptions. Therefore, an assumption was accepted about a subjective character of information. Information is treated as a feature of a cognition process. It is not an objective element of the reality. Furthermore, there was also assumed that uncertainty is a result of interference in mapping information and processes connected with cognition of the reality by human. Starting from these assumptions, the article demonstrates the way uncertainty is modelled and perceived in terms of probability, in fuzzy logic, in rough set theory, and in grey systems theory, which has been gaining popularity in recent years. In order to compare the mentioned approaches to a maximum extent, an attempt was made to show their essence referring to a classical set theory. To this aim, mathematical formalism was used for a precise description of particular types of uncertainty. It is of great significance as in management sciences and generally in social sciences some terms, for example, fuzziness, probability or greyness are overinterpreted and used in descriptive way to pretend scientific statements.
\end{abstract}

Keywords: uncertainty, fuzzy logic, rough set theory, grey systems theory, probability.

\section{UNCERTAINTY AND ITS SIGNIFICANCE IN MANAGEMENT PROCESSES}

When analysing uncertainty issues, a starting point should be the focus on the matters of a cognition process of the reality. It happens because uncertainty is caused by incomplete mapping of the reality in the subject's mind. The mapping appears as a result of complicated information processes pursued by the subject. In the first place, the subject recognises objectively existing states of object. These recognised states can be called data. However, the meaning, which is given to data by the subject of cognition, is called information. Considering the following line of thinking, we can say that data are epistemologically objective, whereas information is of subjective character and it is depended on a subject of cognition.

${ }^{1}$ Rafał Mierzwiak, PhD, Postdoctoral Researcher, Colleague of Economics and Management, Nanjing University of Aeronautics and Astronautics, 211106 Nanjing, 29 Yudao St., P.R. China, tel.: +86 18851879117; Assistant Professor, Faculty of Management Engineering, Poznan University of Technology, 60-965 Poznań, 11 Strzelecka Str., Poland, rafal.mierzwiak@ put.poznan.pl, tel.: +48 691504270. ORCID: 0000-0003-1763-1012. 
The concept of information is frequently treated as a synonym of the concept of knowledge. Hence, these two concepts ought to be differentiated as knowledge is a set of subject's statements based on possessed information. This leads to the following statement, namely information is intentional towards data while knowledge is intentional towards information. Such a distinction is very important for uncertainty modelling as an original cause of uncertainty is uncertainty of possessed information. Accepting this assumption, we can state that methods of uncertainty mathematical modelling are focused on the attempt of grasping a cognitive relation between data and a subject of an information process.

Referring to the assumption about an original character of information processes towards processes of creating knowledge and assuming epistemological subjectivism of information and its direct intentionality towards the reality, we can claim that human as a subject of cognition might create the following types of information concerning an object that is analysed by him (Mierzwiak, Xie, Nowak, 2018):

- only a positive existential statement saying that an object exists without any information about its features and characteristics. In such a case, the level of uncertainty about the object is maximal because the subject does not have any knowledge about its significant quality.

- complete information about an analysed object and its all characteristics. This case of cognition does not entail uncertainty. Obviously, information completeness and fullness are of a relative character because these features are depended on feelings of the subject of cognition.

- partial and incomplete information about the object and its all characteristics. Here, a subject of cognition knows about his cognitive limitations resulting from internal and external conditions.

From the viewpoints of science and practice, the most import case in the context of uncertainty modelling is the one of partial and incomplete information. Incompleteness and partiality of information can refer to the range within which an object is recognised (an identification error), the state of particular characteristics of the object (a scale error), and relations between characteristics in the object (an error of relation).

All the mentioned types of cognitive errors might play significant roles in management processes. It happens due to the fact that management processes can be treated as complex information and decision processes aimed at coordination of subjects' actions, with people included in the subject's structure of purposes and resources.

Thus, on each stage of a decision-making process, managers deal with social subsystems, which an indispensable feature is extreme complexity. This causes the situation where full cognition of those systems is impossible. That is why, the information and decision process pursued by managers always takes place in the conditions of uncertainty. Still, there is a group of routine decisions, in which this uncertainty is minimal, but with the growth of organisation's innovativeness and its drive towards maximizing the dynamic efficiency, the level of uncertainty is rapidly increasing.

Uncertainty phenomenon in management processes enforces the need for newer methods of its modelling and analysis at the operational level. Nowadays, statistical models are commonly used. They have long traditions of their application in management and basically, they fall into a standard series of methods used in this field of knowledge (Aczel, 1993). Another very popular approach is fuzzy logic, especially in modelling the concepts expressed in a natural language (Altrock, 1996). An interesting view in the area of forming 
rules of procedure for managers on the basis of existing incomplete data is given by a rough set theory. Next contemporary developed research stream in uncertainty modelling is grey systems theory more and more popular in economic applications. It found plenty of applications where a researcher holds a small number of data (Pawlak, 2002).

The approaches to uncertainty modelling mentioned above will be presented in the latter part of the article. Their analysis will be carried out from the viewpoint of their usefulness and purposefulness of application in social sciences. Acknowledging that economics and management are social sciences; thus, under the generalisation, all the methodological remarks concerning social sciences will also be applicable to management sciences.

\section{PROBABILISTIC APPROACH TO UNCERTAINTY}

Historically, the oldest and very firmly established way of uncertainty analysis is a probabilistic approach. It is contemporary based on Kolmogarov's axioms and it is connected with a mathematical theory of measure (Kolmogorov, 1950). In reference to this approach, probability is determined with the use of a probabilistic space $\{\Omega, F, P\}$.

Definition 1. Assume that there is a probabilistic space $\{\Omega, F, P\}$ where:

- $\Omega$ is a sample space,

- $F$ is $\sigma$-algebra determined on $\Omega$, provided that a set of subset of $\Omega$ is called $\sigma$-algebra only when $\varnothing \in F$ and $A \in F \Rightarrow A^{\prime} \in F$ and $A_{1}, A_{2} \ldots \in F \Rightarrow \bigcup_{n=1}^{\infty} A_{n}$

- $P$ is a function that ascribes numbers from a set of real numbers to $F$, what formally can be written as follows $P: F \rightarrow R$

Making use of a probabilistic space, it is possible to determine probability in a formal way (see Definition 2).

Definition 2. Function $P: F \rightarrow R$ is called a probability function when it fulfils three axioms such as:

- $P(A) \geq 0$ for any $A \in F$,

- $P(\Omega)=1$,

- $P\left(A_{1} \cup A_{2} \ldots . . A_{n}\right)=P\left(A_{1} \cup A_{2} \ldots A_{n}\right)$ if set are pairwise disjoint i.e. $A_{i} \cap A_{j}=\varnothing$ for $i \neq j$.

Axiomatization of probability is a foundation for a methodological status of statistical methods, which are at present as a basic tool of data analysis.

However, it is not the only interpretation of a probability term known in subject literature. There are a lot of them, and an attempt of their classification might pose many difficulties and arouse controversy. An interesting proposal in this field was presented by Ballentine (Ballentine, 2001). According to him, a concept of probability is a result of accepting inductive reasoning logic (Jaynes, 2003; Cox, 1964; Cox, 1961; Jeffreys, 1973). In a proposed classification, he distinguishes Bernulli's interpretation based on a classical frequency approach (Kolmogorov, 1950; Mises, 1957) and Popper's interpretation (Popper, 1957) that emphasizes propensity as a concept which is a mitigation of the assumption about a deterministic character of the reality. This classification also includes approaches that stress the subjective feeling of a probability category, where it takes the form of commonsense statements about the reality. The commonsense statements are given by a subject of cognition on the grounds of his subjective experience and incomplete knowledge (Finetti, 
1972; Savage 1951; Good, 1983). A classification of Ballentine's different probabilistic approaches in a descriptive way is shown in Figure 1.

A probabilistic approach to uncertainty modelling has found multiple applications. Still, it also has some restrictions of methodological nature. In particular, they were discussed in social sciences, mainly in theoretical economics. It was considered whether a category of probability can be useful in reference to forecasting the structures of purposes, resources, and effects of human actions (Hoppe, 2007; Dow, Hillard, 1995). Probabilistic methods assume, in the majority, established distributions of random variables that describe analysed phenomena. In fact, in problems typical for management and economics, the analysed variables might not meet theoretical assumptions. What is more, a problem of variables' measurement also occurs. Usually in social sciences, we deal with variables which we can measure at most on the ordinal scale. Therefore, a lot of probabilistic methods e.g. all the statistical parametric methods can be used with limited methodological trust as for achieved results.

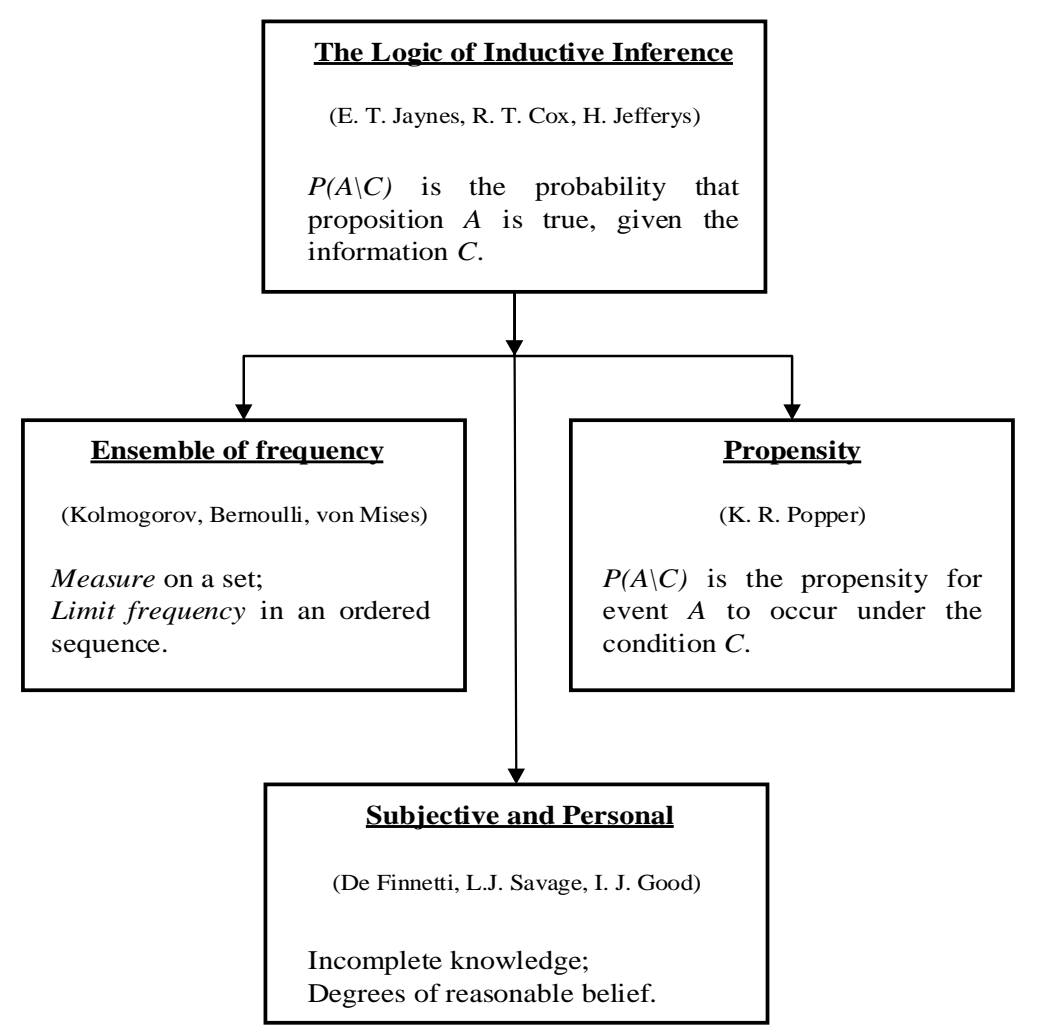

Fig. 1. Classification of probability interpretations

Source: L.E. Ballentine, Interpretations of probability and quantum theory, Foundations of probability and physics, World Scientific Singapore, 2001. 


\section{FUZZY LOGIC IN UNCERTAINTY MODELLING}

Another important theory of uncertainty modelling, which found widespread practical applications in data analysis, is fuzzy logic. It was initiated as a generalisation of a classical set theory (Zadeh, 1965). In a fuzzy approach, elements of the set can belong to the set to some extent, but not definitely as it is in a classical set theory.

Definition 3. A fuzzy set $A$ on a certain space $X$ is called a set of pairs:

$$
A=\left\{\left(x, \mu_{A}\right): x \in X\right\}
$$

where: $\mu_{A} \in[0,1]$ is called a degree of membership of any element $x$ to a set $A$.

If we assume that $\mu_{A}$ reflects the character of a function, then such a function is called a membership function.

Definition 4. Function $\mu_{A}$ that mapps the membership of any element $x$ to a set $A$ is called a memebership function when:

$$
\mu_{A}: X \rightarrow[0,1] \Rightarrow \mu_{A}(x) \in[0,1]
$$

If space $X$, on which any fuzzy set was determined, belongs to a set of real numbers, then we can identyfiy the fuzzy set with a fuzzy number.

Definition 5. A fuzzy number is called such a fuzzy set $A$ that:

$$
A=\left\{\left(x, \mu_{A}\right): x \in X \wedge X=R\right\}
$$

where $R$ means a set of real numbers.

Fuzzy logic has been widely used for analysing uncertainty in social sciences as well as technical sciences (Ragin, 2000; Buckley, Eslami, Feuring, 2013, Vol. 91). It allows to formal modelling of blurred concepts resulting from imprecision of a natural language. Its classical applications are supporting decision-making processes (Zadeh, 1983, 11(1-3)), a mathematical formalization of linguistic variables (Zadeh, 1975, 8(3)), and usage in contribution to control processes (Lee, 1990, 20(2)).

Despite several successful practical applications of fuzzy logic, it is worth to bear in mind its weaknesses. One of the major ones is the fact that a choice of membership functions is arbitrary. Next, carrying out consecutive operations on fuzzy quantities is increasing the level of uncertainty in analysis. Thus, it is extremely important to conduct studies aimed at finding ways to overcome these difficulties and modify the principles of performing fuzzy algebraic operations proposed in a classical version by Zadeh (Kosiński, Prokopowicz, 2016, 32(46/05); Kosiński, Prokopowicz, Ślęzak, 2003, 51(3)).

\section{ROUGH SET THEORY}

A different view on uncertainty, in comparison with a fuzzy logic and a probabilistic approach, can be found in a rough set theory. The theory is a kind of a mathematical formalism, which is useful in modelling fuzzy concepts. According to the main idea of the rough set theory, we possess some information about the universe, and we make use of information when creating the sets. It is a completely different approach than in a classical set theory where sets are determined on the basis of information about the characteristics of elements excluding any knowledge concerning the universe. For a formal description of rough sets, we need a concept of an information system (Pawlak, 1982, 11(5); Pawlak, 2004, (5)). The example of an information system is shown in Figure 2. 


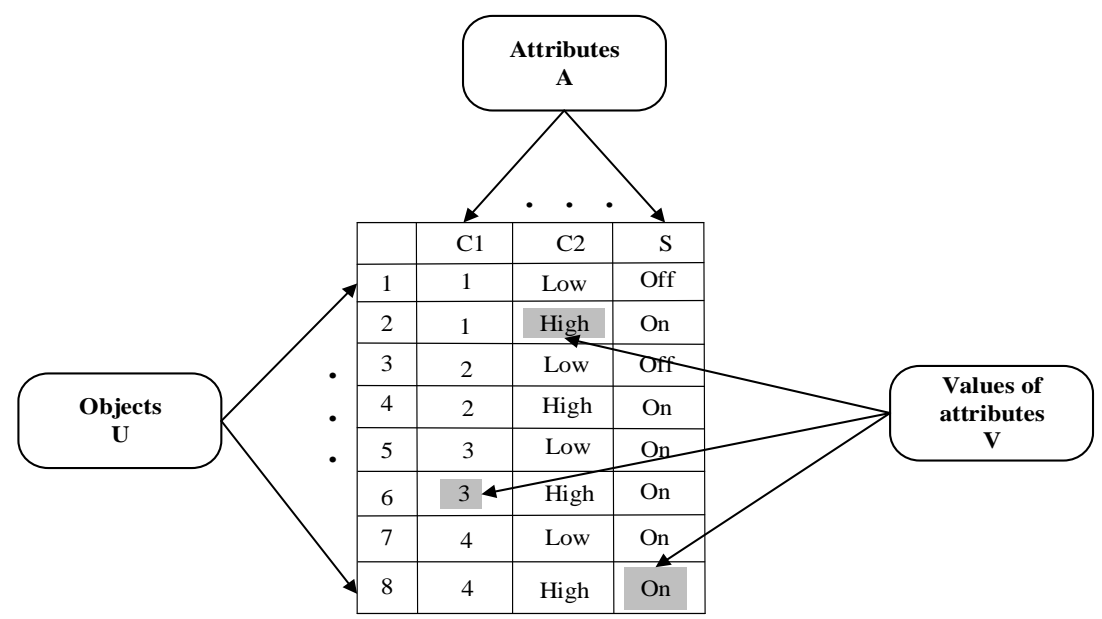

Fig. 2. Example of an information system

Source: Z. Pawlak, Rough sets. New method of data analysis. Warsaw University Magazine, (5) 2004 (in Polish).

In a formal way, the structure of the information system can be presented in the conformation of a four-element set (Pawlak, 2004, (5)).

Definition 6. Information system is called a set of elements:

$$
S I=(U, A, V, f)
$$

where: $U-$ a non-empty finished set of objects called universe,

$A$ - a set of attributes,

$V$ - value of attributes from a set $A$ such that $V=\bigcup_{a \in A} V_{a}$, provided that $V_{a}$ is a field of the attribute $a \in A$,

$f$ - an information function such that $f: U \times A \rightarrow V$, provided that $\forall a \in A$, $x \in U, f(a, x) \in V_{a}$.

Having a formally defined information system, it is possible to combine an indiscernibility relation $I(B)$ with every subset of attributes $B \subseteq A$.

Definition 7. Indiscernibility relation $I(B)$ is called such a relation, which fulfils the following condition:

$$
I(B)=\{(x, y) \in U \times U: \forall a \in B ; f(a, x)=f(a, y)\} B \subseteq A
$$

A set of all classes of $I(B)$ relation's abstractions provides a division of a set $U$ with the use of a set $B$. Assume that $B(x)$ is a class of abstraction of $I(B)$ relation that includes some object $x$. Moreover, let us also assume that we want to determine a non-empty set $X$ which is a subset $U$ in the categories of attributes $B$. It is possible only through defining the two following sets:

$$
B_{*}(X)=\{x \in U: B(x) \subseteq X\} \ldots B^{*}(X)=\{x \in U: B(x) \cap X \neq \varnothing\}
$$

$B_{*}(X)$ is a lower approximation of a set and it contains objects, which definitely can be included in a set $X$; whereas $B^{*}(X)$ is an upper approximation of a set where objects can be 
only regarded as possibly belonging to a set $X$. Differences between $B_{*}(X)$ and $B^{*}(X)$ are called a boundary of a set $X$ and we write it down as: $B N_{B}(X)=B^{*}(X)-B_{*}(X)$. A boundary $B N_{B}(X)$ includes objects, which unambigiously cannot be ascribed to $X$ due to a contradictory description in attributes. However, objcets that belong to $U \backslash B N_{B}(X)$ definitely can be regarded as the ones which do not belong to $X$. Such an idea is presented in Figure 3 in the descriptive way.

The concept of rough sets can be applied in describing imprecise knowledge about a depicted phanomenon. On their basis, it is possible to generate principles that map interdependences which take place in data. Still, a rough set theory does not replace a classical set approach. It is only its supplement. Contrary to a fuzzy approach, a rough set theory is based on the concept of indiscernibility of elements, which is depended on existing empirical material. Thus, indiscernibility is not established in an arbitrary way as it is in case of a membership function in fuzzy logic. On the one hand, it is an advantage, which involves objectification of analysis through direct turning to data. On the other hand, it might cause lower flexibility in applications (Tadeusiewicz, access: 17th October 2017).

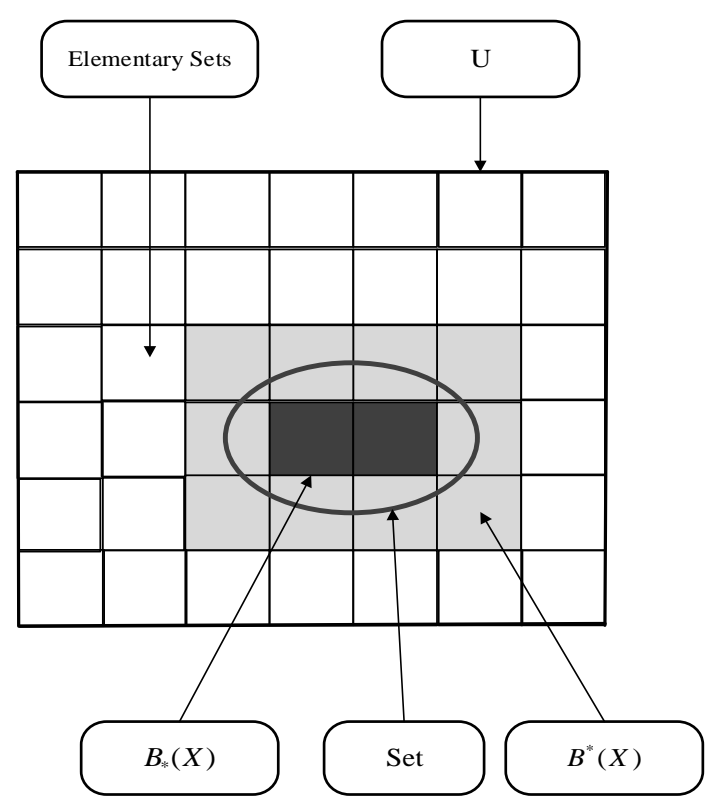

Fig. 3. Lower and upper approximation of a set.

Source: Z. Pawlak, Rough sets. New method of data analysis. Warsaw University Magazine, (5) 2004 (in Polish).

\section{GREY SYSTEMS THEORY}

Grey systems theory (GST) is a relatively new developed theory of uncertainty modelling compared with statistics or fuzzy logic. Similarly to a rough set theory, it started in the early 1980s; however, due to a lack of English-language works its popularity and development in international environment was very limited. Only at the beginning of the 
1990s the first more important publications in English language began to appear. Only then, its wider popularisation in the scientific community was possible (Lin, Liu, 2004).

In order to describe the reality, GST uses the following general axioms concerning the nature of information (Liu, Yang, Forrest, 2016):

- Axiom of Informational Differences. "Difference" implies the existence of information. Each piece of information must carry some kind of "difference".

- Axiom of Non-Uniqueness. The solution to any problem with incomplete and indeterminate information is not unique.

- Axiom of Minimal Information. One characteristic of grey system theory is that it makes the most and best use of the "minimal amount of available information."

- Axiom of Recognition Base. Information is the foundation on which people recognize and understand (nature).

- Axiom of New Information Priority. The function of new pieces of information is greater than that of old pieces of information.

- Axiom of Absolute Greyness. "Incompleteness" of information is absolute. Incompleteness and non-determinism of information have generality.

The principles demonstrated above are not axioms in the strict sense. They are more like philosophical assumptions connected with the nature of a cognition process. On their basis, it is not possible to either strictly define a concept of grey information or determine forms of its expression in a logical way (Mierzwiak, Xie, Nowak, 2018, 8(2)). However, it can be done through creating so called a grey space that consists of three elements which are sets (Mierzwiak, et. al, 2018).

Definition 8. Assume that we have the following grey space $\left\{\Omega, F, G^{\circ}\right\}$. In this space:

- $\Omega$ means a set, which is a space for analysis where all the other sets in this analysis belong to,

- $F$ is $\sigma$-algebra generated on a set $\Omega$ i.e. $\varnothing \in F$ and $A \in F \Rightarrow A^{\prime} \in F$ and $A_{1}, A_{2} \ldots A_{i} \in F \Rightarrow \bigcup_{i=1}^{\infty} A_{i} \in F$

- $G^{\circ}$ is a function mapping $F$ in the set of real numbers, what formally can be written down as follows $G^{\circ}: F \rightarrow R$, and it is a function of greyness when it fulfils such axioms as:

1) $G^{\circ}\left(A_{i}\right) \geq 0$ for every $A_{i} \in F$,

2) $G^{\circ}\left(A_{i}\right)=0 \Leftrightarrow A_{i}=\left\{a_{i}\right\}$, where $A_{i}=\left\{a_{i}\right\}$ means any singleton belonging to $F$,

3) $G^{\circ}(\Omega)=1$,

4) $G^{\circ}\left(\bigcup_{i \in N} A_{i}\right)=\sum_{i \in N} G^{\circ}\left(A_{i}\right), \quad$ where $\quad A_{i} \cap A_{j}=\varnothing \wedge i \neq j \wedge A_{i} \neq\left\{a_{i}\right\} \quad$ or $G^{\circ}\left(\bigcup_{i \in N} A_{i}\right)=G^{\circ}(A)$, where $A_{i} \cap A_{j}=\varnothing \wedge i \neq j \wedge A_{i}=\left\{a_{i}\right\} \wedge A=\bigcup_{i \in N} A_{i}$,

Referring to a grey space, all important basic categories of grey systems theory can be generalised. One of the most significant categories of these types are grey numbers. A grey number is a number, which value is not exactly known. Only an interval in which this value is placed is known. These numbers might be used for the analysis of problems about which we only know the order of an analysed magnitude. 
Definition 9. If $\left\{\Omega, F, G^{\circ}\right\}$ is a grey space and $\Omega \subset R$ and $D \in F$, then a grey number $\otimes=[a, b]$ is called an unknown number $d^{*} \in R$ about which we can only say that $a \leq d^{*} \leq b$, where $a=\inf (D)$ and $b=\sup (D)$.

Apart from grey numbers, a major construct used in GST is a weight function of whitenization. This function is significant in many methods that support decision-making and evaluation processes.

Definition 10. Assume that a grey space $\left\{\Omega, F, G^{\circ}\right\}$ and a set $A \in F$ and a set $Y=$ $=\{y \in R: 0 \leq y \leq 1\}$, then a function $f: A \rightarrow Y$ is called a weight function of whitenization if it additionally preserves the following conditions:

1) $f(A)=1$ for every $A=\{a\}$, what means for every singleton

2) $f(\varnothing)=0$.

A crucial element of grey systems theory is an analysis of data sequences. The sequences can concern time, information about the state of evaluation criteria of some analysed object or information about a set of objects. Data in a sequence are not expressed with the use of grey numbers, because greyness of information is ascribed to the whole data set in the sequence, not to particular elements that constitute this sequence. Making use of a grey space, we can strenghten this way of thinikng, which has a fundamental meaning for GST applications in terms of a relation analysis between variables and forecasting (Mierzwiak, et. al, 2018).

Currently GST has found a lot of applications in various branches of science and business practice, especially in economics and management (Delcea, 2014, 26(1); Delcea, 2015, $5(2)$ ). Basically, it can be regarded as one of the techniques of data mining, which has a specific scope of applications connected with a small number of data. Except for statistics, fuzzy logic or a rough set theory, it can be another methodology of uncertain data analysis. Restrictions of using GST in economic and management problems are the same as the restrictions in all mathematical methods of uncertainty modelling, i.e. they achieve satisfactory results in statistical problems. However, when it comes to problems of a dynamic structure of resources and action purposes, these methods do not bring the desirable results. Furthermore, the attention might be paid to the fact that forecasting methods in GST are trying to predict accurately states of the analysed systems in coordinates of time. While it makes perfect sense in case of technical issues, it arouses serious methodological controversy in the event of economic systems (De Soto, 1998). Another essential issue is drawing conclusions from the analysis on the basis of a small number of data. GST methods are constructed in such a way that a small number of data is not an obstacle in an analytical process. Still, it should be noticed that in the induction process and generalisation of results, a method of analysis does not matter as much as the representativeness of a research sample. Therefore, the conclusions arising from analyses carried out with GST ought to be treated very carefully.

\section{CONCLUSION}

Currently, correct data analysis and appropriate dealing with an uncertainty problem have started to be meaningful for many organisations (Pietrewicz, 22 24(2)). Development of information techniques allows to handle the calculation complexity of analytical algorithms and large data sets. In the following article, basic approaches to uncertainty modelling were analysed such as probability, fuzzy logic, a rough set theory, and a grey systems theory. Strengths and weaknesses of each approach were indicated in the context of 
management and economic sciences, and conclusions and recommendations were formulated. An extremely important conclusion for a management practice is that demonstrated methods of mathematical modelling in regard to the organisations aiming at achieving a dynamic efficiency might not be of much value. Thus, this concerns all the organisations, which are directed towards creativity and innovations. On the other hand, good results of mathematical methods can be obtained as for modelling of the organisation with a stabilised structure, purposes, and action resources; therefore, in case of the organisations striving for maximising a static efficiency.

Further studies should be related to a systematic analysis of literature in the area of applications of particular approaches to uncertainty modelling. Very interesting might be the results of such an analysis in reference to a grey systems theory, because it is the least known and popular. What is more, carrying out this analysis can indicate main tendencies in a given scientific discipline and identify new research gaps, which has been unknown so far, on the borderline of management sciences and data science.

\section{REFERENCES}

Aczel, A.D. (1993). Complete business statistics. Irwin Professional Publishing.

Altrock, C. (1996). Fuzzy logic and neurofuzzy applications in business and finance. Prentice-Hall, Inc.

Buckley, J.J., Eslami, E., Feuring, T., (2013). Fuzzy mathematics in economics and engineering. "Physica" Vol. 91.

Ballentine, L.E. (2001). Interpretations of probability and quantum theory. Foundations of probability and physics, World Scientific Singapore.

Cox, R.T. (1964). Probability, frequency and reasonable expectation. "American Journal of Physics" 14(1).

- (1961). Algebra of Probable Inference. JHU Press.

de Finetti, B. (1972). Probability, induction, and statistics. New York: Wiley.

Delcea, C. (2014). Not black not even white definitively grey economic systems. "The Journal of Grey System” 26(1).

- (2015). Grey systems theory in economics - a historical applications review. Grey Systems: Theory and Application, 5(2).

De Soto, J.H. (1998). The ongoing methodenstreit of the Austrian school. Centre d'analyse économique.

Dow, S., Hillard, J. (1995). Keynes, knowledge and uncertainty. Edward Elgar Publishing.

Good, I.J. (1983). Good thinking: The foundations of probability and its applications. University of Minnesota Press.

Hoppe, H.H. (2007). The limits of numerical probability: Frank H. Knight and Ludwig von Mises and the frequency interpretation. "Quarterly Journal of Austrian Economics" 10(1).

Jaynes, E.T. (2003). Probability theory: The logic of science. Cambridge University Press.

Jeffreys, H. (1973). Scientific inference. Cambridge University Press.

Kolmogorov, A.N. (1950). Foundations of probability theory. Chelsea, New York.

Kosiński, W., Prokopowicz, P. (2016). Algebra of fuzzy numbers. "Mathematica Applicanda" 32(46/05).

Kosiński, W., Prokopowicz, P., Ślęzak, D. (2003). Ordered fuzzy numbers. "Bulletin of the Polish Academy of Sciences, Series Science Mathematica” 51(3). 
Lee, C.C. (1990). Fuzzy logic in control systems: fuzzy logic controller. IEEE Transactions on systems, man, and cybernetics, 20(2).

Mierzwiak, R., Xie, N., Nowak, M. (2018). New axiomatic approach to the concept of grey information. Grey Systems: Theory and Application, 8(2).

Mises, R. (1957). Probability, statistics, and truth. Courier Corporation.

Pawlak, Z. (2002). Rough sets and intelligent data analysis. "Information sciences" 147(1-4).

- (2004). Rough sets. New method of data analysis. "Warsaw University Magazine”, Vol. 5 (in Polish).

(1982). Rough sets. "International Journal of Parallel Programming” 11(5).

Pietrewicz, L. Uncertainty, postmodernism and economic growth. "Humanities and Social Sciences", Vol. 2224 (2) (in Polish).

Popper, K.R. (1957). The propensity interpretation of the calculus of probability, and the quantum theory [In:] Körner, S. (ed.), Observation and Interpretation, Butterworths.

Lin, Y., Liu, S. (2004). A historical introduction to grey systems theory, IEEE International Conference: Systems, Man and Cybernetics.

Liu, S., Yang, Y., Forrest, J. (2016). Grey Data Analysis: Methods, Models and Applications. Springer, 2016.

Ragin, C.C. (2000). Fuzzy-set social science. University of Chicago Press.

Savage, L.J. (1951). The theory of statistical decision. "Journal of the American Statistical Association" 46(253).

Tadeusiewicz, R. (2017). Polish Island in archipelago of Artificial Intelligence - rough sets. [Access: 17.10.2017]. Access on the internet: http://ryszardtadeusiewicz.natemat.pl/150285, polska-wyspa-w-archipelagu-sztucznej-inteligencji-zbiory-przyblizone

Zadeh, L.A. (1965). Fuzzy sets. „Information and control” 8(3).

- (1975). The concept of a linguistic variable and its application to approximate reasoning. "Information sciences" 8(3).

— (1983). The role of fuzzy logic in the management of uncertainty in expert systems. "Fuzzy sets and systems" 11(1-3).

DOI: $10.7862 /$ rz.2019.hss.7

The text was submitted to the editorial office: June 2018.

The text was accepted for publication: March 2019. 
